\title{
HIGH-ENERGY GAMMA RAYS FROM STELLAR ASSOCIATIONS
}

\author{
Diego F. Torres, ${ }^{1}$ Eva Domingo-Santamaría, ${ }^{2}$ and Gustavo E. Romero ${ }^{3}$ \\ Received 2003 October 22; accepted 2003 December 4; published 2004 January 16
}

\begin{abstract}
It is proposed that $\mathrm{TeV} \gamma$-rays and neutrinos can be produced by cosmic rays (CRs) through hadronic interactions in the innermost parts of the winds of massive $\mathrm{O}$ and $\mathrm{B}$ stars. Convection prevents low-energy particles from penetrating into the wind, leading to an absence of $\mathrm{MeV}-\mathrm{GeV}$ counterparts. It is argued that groups of stars located close to the CR acceleration sites in OB stellar associations may be detectable by ground-based Cerenkov telescopes.
\end{abstract}

Subject headings: gamma rays: observations — gamma rays: theory — stars: early-type

\section{INTRODUCTION}

Several $\gamma$-ray sources are thought to be related to early-type stars and their neighborhoods (e.g., Montmerle 1979; Cassé \& Paul 1980; Bykov \& Fleishman 1992a, 1992b; Bykov 2001, Romero \& Torres 2003). Recently, the first (and only) TeV unidentified source was detected in the Cygnus region (Aharonian et al. 2002), where a nearby EGRET source (3EG J2033 + 4118) has a likely stellar origin (White \& Chen 1992; Chen, White, \& Bertsch 1996; Romero, Benaglia, \& Torres 1999; Benaglia et al. 2001). Here, we explore whether cosmicray $(\mathrm{CR})$ illumination of stellar winds of $\mathrm{O}$ and $\mathrm{B}$ stars can lead to Galactic TeV $\gamma$-ray sources.

\section{THE MODEL}

$\mathrm{O}$ and $\mathrm{B}$ stars lose a significant fraction of their mass in stellar winds with terminal velocities $V_{\infty} \sim 10^{3} \mathrm{~km} \mathrm{~s}^{-1}$. With mass-loss rates as high as $\dot{M}_{*}=10^{-6}$ to $10^{-4} M_{\odot} \mathrm{yr}^{-1}$, the density at the base of the wind can reach $10^{-12} \mathrm{~g} \mathrm{~cm}^{-3}$ (e.g., Lamers \& Cassinelli 1999, p. 8). Such winds are permeated by significant magnetic fields and provide a matter field dense enough as to produce hadronic $\gamma$-ray emission when pervaded by relativistic particles. A typical wind configuration (Castor, McCray, \& Weaver 1975; Völk \& Forman 1982; Lamers \& Cassinelli 1999, p. 355) contains an inner region in free expansion (zone 1) and a much larger hot compressed wind (zone $2)$. These are finally surrounded by a thin layer of dense sweptup gas (zone 3) - the final interface with the interstellar medium (ISM). The innermost region size is fixed by requiring that at the end of the free expansion phase (about $100 \mathrm{yr}$ after the wind turns on), the swept-up material is comparable to the mass in the driven wave from the wind, which happens at a radius $R_{\text {wind }}=V_{\infty}\left(3 \dot{M}_{*} / 4 \pi \rho_{0} V_{\infty}^{3}\right)^{1 / 2}$, where $\rho_{0} \approx m_{p} n_{0}$ is the ISM mass density, with $m_{p}$ the mass of the proton and $n_{0}$ the ISM number density. After hundreds of thousands of years, the wind produces a bubble with a radius of the order of tens of parsecs, with a density lower (except that in zone 1) than in the ISM. In what follows, we consider the hadronic production of $\gamma$-rays in zone 1 , the innermost and densest region of the wind. The matter there is described through the continuity equation, $\dot{M}_{*}=4 \pi r^{2} \rho(r) V(r)$, where $\rho(r)$ is the density of the wind and $V(r)=V_{\infty}\left(1-R_{0} / r\right)^{\beta}$ is its velocity. Here $V_{\infty}$ is the terminal

\footnotetext{
${ }^{1}$ Lawrence Livermore National Laboratory, 7000 East Avenue, L-413, Livermore, CA 94550; dtorres@igpp.ucllnl.org.

${ }^{2}$ Institut de Física d'Altes Energies (IFAE), Edifici C-n, Campus UAB, 08193 Bellaterra, Spain; domingo@ifae.es.

${ }^{3}$ Instituto Argentino de Radioastronomía (IAR), C.C. 5, 1894 Villa Elisa, Argentina; romero@irma.iar.unlp.edu.ar.
}

wind velocity, and the parameter $\beta$ is $\sim 1$ for massive stars (Lamers \& Cassinelli 1999, p. 8). $R_{0}$ is given in terms of the wind velocity close to the star, $V_{0} \sim 10^{-2} V_{\infty}$, as $R_{0}=R_{*}[1-$ $\left.\left(V_{0} / V_{\infty}\right)^{1 / \beta}\right]$. Hence, the particle density is $n(r)=\dot{M}_{*}(1-$ $\left.R_{0} / r\right)^{-\beta} /\left(4 \pi m_{p} V_{\infty} r^{2}\right)$.

Not all CRs will enter into the base of the wind. Although wind modulation has been studied in detail only for the case of the relatively weak solar wind (e.g., Parker 1958; Jokipii \& Parker 1970; Kóta \& Jokipii 1983; Jokipii, Kóta, \& Merényi 1993), a first approach to determine whether particles can pervade the wind is to compute the ratio $(\epsilon)$ between the diffusion and convection timescales: $t_{d}=3 r^{2} / D$ and $t_{c}=3 r / V(r)$, where $D$ is the diffusion coefficient and $r$ is the position in the wind. Only particles for which $\epsilon<1$ will be able to overcome convection and enter the dense wind region to produce $\gamma$-rays through $p-p$ interactions. The diffusion coefficient is $D \sim$ $\lambda_{r} c / 3$, where $\lambda_{r}$ is the mean free path for diffusion in the radial direction. As in White (1985) and Völk \& Forman (1982), the mean free path for scattering parallel to the magnetic field $(B)$ direction is assumed as $\lambda_{\|} \sim 10 r_{g}=10 E / e B$, where $r_{g}$ is the particle gyroradius and $E$ its energy. In the perpendicular direction $\lambda$ is shorter, $\lambda_{\perp} \sim r_{g}$. The mean free path in the radial direction is then given by $\lambda_{r}=\lambda_{\perp} \sin ^{2} \theta+\lambda_{\|} \cos ^{2} \theta=r_{g}\left(10 \cos ^{2} \theta+\right.$ $\left.\sin ^{2} \theta\right)$, where $\cos ^{-2} \theta=1+\left(B_{\phi} / B_{r}\right)^{2}$. Here, the geometry of the magnetic field is represented by the magnetic rotator theory (Weber \& Davis 1967; White 1985; Lamers \& Cassinelli 1999, p. 255) $B_{\phi} / B_{r}=\left(V_{*} / V_{\infty}\right)\left(1+r / R_{*}\right)$ and $B_{r}=B_{*}\left(R_{*} / r\right)^{2}$, where $V_{*}$ is the rotational velocity at the surface of the star and $B_{*}$ the surface magnetic field. Using all previous formulae, $\epsilon \sim\left(3 e B_{*} V_{\infty}(r-\right.$ $\left.\left.R_{*}\right)\left(R_{*} / r\right)^{2}\left\{1+\left[V_{*} / V_{\infty}\left(1+r / R_{*}\right)\right]^{2}\right\}^{3 / 2}\right) /\left(E_{p} c\left\{10+\left[V_{*} / V_{\infty}(1+\right.\right.\right.$ $\left.\left.\left.\left.r / R_{*}\right)\right]^{2}\right\}\right)$. The latter equation defines a minimum energy $E_{p}^{\text {min }}(r)$ below which the particles are convected away from the wind (shown in Fig. 1, left). Note that $E_{p}^{\min }(r)$ is an increasing function of $r$, so particles that are not convected away in the outer regions of the wind are able to diffuse up to its base. Then $E_{p}^{\min }(r)$ can be effectively approximated by $E_{p}^{\min }(r \gg$ $R_{*}$ ) in subsequent computations. Only particles with energies higher than a few $\mathrm{TeV}$ will interact with nuclei in the inner wind and ultimately generate $\gamma$-rays, substantially reducing the flux in the MeV-GeV band.

The opacity to pair production of the $\gamma$-rays in the UV stellar photon field can be computed as $\tau\left(R_{c}, E_{\gamma}\right)=$ $\int_{0}^{\infty} \int_{R_{c}}^{\infty} N\left(E_{*}\right) \sigma_{e^{-} e^{+}}\left(E_{*}, E_{\gamma}\right) d E_{*} d r$, where $E_{*}$ is the energy of the photons emitted by the star, $E_{\gamma}$ is the energy of the $\gamma$-ray, $R_{c}$ is the place where the photon was created within the wind, and $\sigma_{e^{-} e^{+}}\left(E_{*}, E_{\gamma}\right)$ is the cross section for $\gamma \gamma$ pair production (Cox 1999 , p. 214). The stellar photon distribution is that of a blackbody peaking at typical star effective temperatures $\left(T_{\text {eff }}\right)$, 

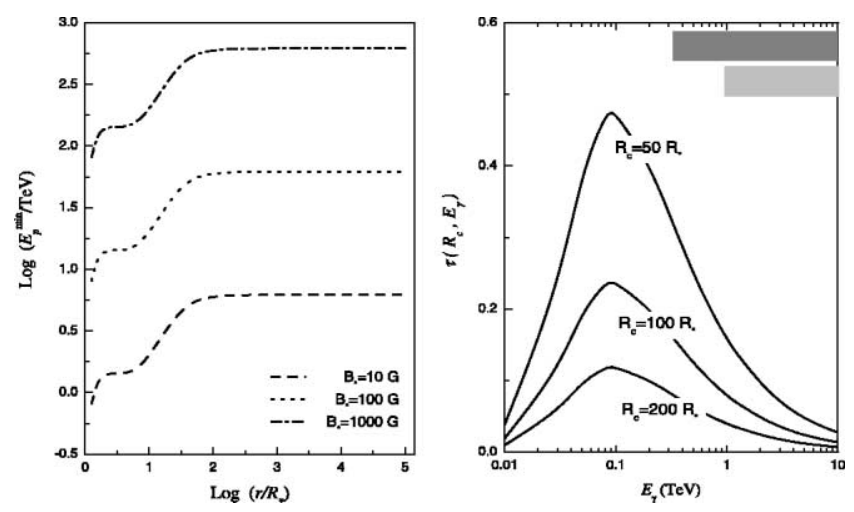

FIG. 1.-Left: Minimum proton energy needed to overcome the wind convection at different distances from the star. Here $V_{*}=250 \mathrm{~km} \mathrm{~s}^{-1}, V_{\infty}=$ $1750 \mathrm{~km} \mathrm{~s}^{-1}$, and $R_{*}=12 R_{\odot}$. Right: Opacities to pair production as a function of the $\gamma$-ray energy for different creation places $R_{c}$. Here, the star has $T_{\text {eff }}=38,000 \mathrm{~K}$.

$N\left(E_{*}\right)=\left[\pi B\left(E_{*}\right) / h E_{*} c\right] R_{*}^{2} / r^{2}$, where $h$ is the Planck constant and $B\left(E_{*}\right)=\left[2 E_{*}^{3} /(h c)^{2}\right] /\left(e^{E_{*} / k T_{\text {eff }}}-1\right)$. Shown in Figure 1 (right) is $\tau\left(R_{c}, E_{\gamma}\right)$ for different photon creation sites $\left(R_{c} \ll\right.$ $R_{\text {wind }}$ ). $\gamma$-ray photons of $\mathrm{TeV}$ and higher energies do not encounter significant opacities in their way out of the wind, unless they are created at its very base, hovering over the star (which is unlikely to happen because $R_{\text {wind }} \gg R_{*}$ and the proton propagates in a high magnetic field environment).

Although we show the opacity for values of the photon energy as low as $100 \mathrm{GeV}$, most of the $\gamma$-rays will have higher energies, since only protons with $E_{p}>E_{p}^{\text {min }}$ will enter the wind. The grey (light grey) box in the figure shows typical energies of $\gamma$-rays for the case of a surface magnetic field $B_{*}=10 \mathrm{G}$ $(100 \mathrm{G})$. There is a large uncertainty about the typical values for the magnetic field in the surface of $\mathrm{O}$ and $\mathrm{B}$ stars, but recent measurements favor $B_{*} \gtrsim 100 \mathrm{G}$ (e.g., Donati et al. 2001, 2002).

\section{GAMMA-RAY AND NEUTRINO EMISSION}

The differential $\gamma$-ray emissivity from $\pi^{0}$-decays can be approximated by $q_{\gamma}\left(E_{\gamma}\right)=4 \pi \sigma_{p p}\left(E_{p}\right)\left(2 Z_{p \rightarrow \pi^{0}}^{(\alpha)} / \alpha\right) J_{p}\left(E_{\gamma}\right) \eta_{A} \Theta\left(E_{p}-\right.$ $\left.E_{p}^{\min }\right)$ at the energies of interest. The parameter $\eta_{A}$ takes into account the contribution from different nuclei in the wind (for a standard composition $\eta_{A} \sim 1.5$; Dermer 1986). $J_{p}\left(E_{\gamma}\right)$ is the proton flux distribution evaluated at $E=E_{\gamma}$ (units of protons per unit time, solid angle, energy band, and area). The cross section $\sigma_{p p}\left(E_{p}\right)$ for $p$ - $p$ interactions at energy $E_{p} \approx 10 E_{\gamma}$ can be represented above $E_{p} \approx 10 \mathrm{GeV}$ by $\sigma_{p p}\left(E_{p}\right) \approx 30[0.95+$ $\left.0.06 \log \left(E_{p} / \mathrm{GeV}\right)\right]$ mbarn (e.g., Aharonian \& Atoyan 1996). $Z_{p \rightarrow \pi^{0}}^{(\alpha)}$ is the so-called spectrum-weighted moment of the inclusive cross section. Its value for different spectral indices $\alpha$ is given, for instance, by Drury, Aharonian, \& Völk (1994). Finally, $\Theta\left(E_{p}-E_{p}^{\mathrm{min}}\right)$ is a Heaviside function that takes into account the fact that only CRs with energies higher than $E_{p}^{\min }\left(r \gg R_{*}\right)$ will diffuse into the wind. The spectral $\gamma$-ray intensity (photons per unit time and energy band) is $I_{\gamma}\left(E_{\gamma}\right)=\int n(r) q_{\gamma}\left(E_{\gamma}\right) d V$, where $V$ is the interaction volume. The luminosity in a given band is $L_{\gamma}=\int_{R_{*}}^{R_{\text {wind }}} \int_{E_{1}}^{E_{2}} n(r) q_{\gamma}\left(E_{\gamma}\right) E_{\gamma}\left(4 \pi r^{2}\right) d r d E_{\gamma}$ (see Torres et al. 2003 and Romero et al. 2003 for details). Assuming a canonical spectrum for the relativistic CR population, $J_{p}\left(E_{p}\right)=(c / 4 \pi) \times$ $N\left(E_{p}\right)=(c / 4 \pi) K_{p} E_{p}^{-\alpha}$, the result (in the range $E_{\gamma} \sim 1-$ $20 \mathrm{TeV}$ ) can be expressed in terms of the normalization $K_{p}$ and will depend on all other model parameters, mainly on the proton (photon) spectral index, the ISM density, the terminal
TABLE 1

EXAMPLES FOR HADRONIC $\gamma$-RAY LUMINOSITIES FROM TyPICAL Stellar Wind Configurations

\begin{tabular}{lccccccc}
\hline \hline & $\begin{array}{c}V_{\infty} \\
\text { Model } \\
\left(\mathrm{km} \mathrm{s}^{-1}\right)\end{array}$ & $\begin{array}{c}n_{0} \\
\left(\mathrm{~cm}^{-3}\right)\end{array}$ & $\begin{array}{c}R_{\text {wind }} \\
(\mathrm{pc})\end{array}$ & $\begin{array}{c}M_{\text {wind }} \\
\left(M_{\odot}\right)\end{array}$ & $\begin{array}{l}L_{\gamma}^{\alpha=1.9} / K_{p} \\
(\mathrm{ergs} \mathrm{s})\end{array}$ & $\begin{array}{c}L_{\gamma}^{\alpha=2.0} / K_{p} \\
\left.(\mathrm{ergs} \mathrm{s})^{-1}\right)\end{array}$ & $\begin{array}{c}L_{\gamma}^{\alpha=2.1} / K_{p} \\
(\mathrm{ergs} \mathrm{s})\end{array}$ \\
\hline $\mathrm{a} \ldots \ldots$ & 1750 & 10 & 0.07 & 0.0004 & $2 \times 10^{28}$ & $7 \times 10^{26}$ & $3 \times 10^{25}$ \\
$\mathrm{~b} \ldots \ldots$ & $\ldots$ & 1 & 0.24 & 0.0013 & $5 \times 10^{28}$ & $2 \times 10^{27}$ & $8 \times 10^{25}$ \\
$\mathrm{c} \ldots \ldots$ & $\ldots$ & 0.1 & 0.75 & 0.0041 & $2 \times 10^{29}$ & $7 \times 10^{27}$ & $3 \times 10^{26}$ \\
$\mathrm{~d} \ldots \ldots$ & $\ldots$ & 0.01 & 2.4 & 0.0130 & $5 \times 10^{29}$ & $2 \times 10^{28}$ & $8 \times 10^{26}$ \\
$\mathrm{e} \ldots \ldots$ & 1000 & 10 & 0.09 & 0.0009 & $4 \times 10^{28}$ & $1 \times 10^{27}$ & $6 \times 10^{25}$ \\
$\mathrm{f} \ldots \ldots$ & $\ldots$ & 1 & 0.31 & 0.0030 & $1 \times 10^{29}$ & $5 \times 10^{27}$ & $2 \times 10^{26}$ \\
$\mathrm{~g} \ldots \ldots$ & $\ldots$ & 0.1 & 0.99 & 0.0095 & $4 \times 10^{29}$ & $1 \times 10^{28}$ & $6 \times 10^{26}$ \\
$\mathrm{~h} \ldots \ldots$ & $\ldots$ & 0.01 & 3.1 & 0.0301 & $1 \times 10^{30}$ & $5 \times 10^{28}$ & $2 \times 10^{27}$ \\
$\mathrm{i} \ldots \ldots$ & 800 & 10 & 0.11 & 0.0013 & $5 \times 10^{28}$ & $2 \times 10^{27}$ & $9 \times 10^{25}$ \\
$\mathrm{j} \ldots \ldots$ & $\ldots$ & 1 & 0.35 & 0.0042 & $1 \times 10^{29}$ & $7 \times 10^{27}$ & $3 \times 10^{26}$ \\
$\mathrm{k} \ldots \ldots$ & $\ldots$ & 0.1 & 1.1 & 0.0133 & $5 \times 10^{29}$ & $2 \times 10^{28}$ & $9 \times 10^{26}$ \\
$1 \ldots \ldots$ & $\ldots$ & 0.01 & 3.5 & 0.0421 & $1 \times 10^{30}$ & $7 \times 10^{28}$ & $3 \times 10^{27}$ \\
\hline
\end{tabular}

velocity, and the mass-loss rate. Very mild dependencies appear with $\beta$ and $R_{*}$. Table 1 presents results for the luminosity for typical values of all these parameters. We have fixed $M_{*}=$ $10^{-5} M_{\odot} \mathrm{yr}^{-1}, \beta=1$, and $R_{*}=12 R_{\odot}$ in this example. The mass contained in the innermost region of the wind, $M_{\text {wind }}$, is also shown. $L_{\gamma} \sim 10^{25}-10^{30} K_{p}$ ergs $\mathrm{s}^{-1}$ can be obtained as the luminosity produced by one particular star; the total luminosity of a group of stars should add contributions from all illuminated winds. Convolving the previous integral with the probability of escape (obtained through the opacity as $e^{-\tau}$ ) does not noticeably change these results. Finally, it is possible to factor out the normalization in favor of the CR enhancement in the region where the wind is immersed. The CR energy density is $\omega_{\mathrm{CR}}=\int N_{p}\left(E_{p}\right) E_{p} d E_{p}=9.9 K_{p} \mathrm{eV} \mathrm{cm}{ }^{-3} \equiv \varsigma \omega_{\mathrm{CR}, \odot}$, where $\varsigma$ is the enhancement factor of the $\mathrm{CR}$ energy density with respect to the local value, $\omega_{\mathrm{CR}, \odot}$ (energies between $1 \mathrm{GeV}$ and $20 \mathrm{TeV})$. Then, $K_{p} \sim(0.2-0.3)$ s.

The $\nu_{\mu}+\bar{\nu}_{\mu}$ neutrino flux $\left[F_{\nu}\left(E_{\nu}\right)\right]$ will be derived from the observed $\gamma$-ray flux $\left[F_{\gamma}\left(E_{\gamma}\right)\right]$ by imposing energy conservation (see Alvarez-Muñiz \& Halzen 2002 for details): $\int E_{\gamma} F_{\gamma}\left(E_{\gamma}\right) d E_{\gamma}=C \int E_{\nu} F_{\nu}\left(E_{\nu}\right) d E_{\nu}$, where the limits of the integrals are $E_{\gamma[\nu]}^{\min }\left(E_{\gamma[\nu]}^{\max }\right)$, the minimum (maximum) energy of the photons [neutrinos], and the prefactor $C$ is a numerical constant of the order of one. Using the resulting $\nu$-flux, the signal for the detection of $\nu$-events can be approximated as (Anchordoqui et al. 2003) $S=T_{\text {obs }} \int d E_{\nu} A_{\text {eff }} F_{\nu}\left(E_{\nu}\right) P_{\nu \rightarrow \mu}\left(E_{\nu}\right)$, whereas the noise will be given by $N=\left[T_{\text {obs }} \int d E_{\nu} A_{\text {eff }} F_{B}\left(E_{\nu}\right) P_{\nu \rightarrow \mu}\left(E_{\nu}\right) \Delta \Omega\right]^{1 / 2}$, where $\Delta \Omega$ is the solid angle of the search bin $\left(\Delta \Omega_{1^{\circ} \times 1^{\circ}} \approx\right.$ $3 \times 10^{-4}$ sr for the ICECUBE telescope; Karle 2002) and $F_{B}\left(E_{\nu}\right) \leqq 0.2\left(E_{\nu} / \mathrm{GeV}\right)^{-3.21} \mathrm{GeV}^{-1} \mathrm{~cm}^{-2} \mathrm{~s}^{-1} \mathrm{sr}^{-1}$ is the $\nu_{\mu}+\bar{\nu}_{\mu}$ atmospheric $\nu$-flux (Volkova 1980; Lipari 1993). Here, $P_{\nu \rightarrow \mu}\left(E_{\nu}\right) \approx 3.3 \times 10^{-13}\left(E_{\nu} / \mathrm{GeV}\right)^{2.2}$ denotes the probability that a $\nu$ of energy $E_{\nu} \sim 1-10^{3} \mathrm{GeV}$, on a trajectory through the detector, produces a $\mu$ (Gaisser, Halzen, \& Stanev 1995). Here $T_{\text {obs }}$ is the observing time and $A_{\text {eff }}$ the effective area of the detector. Those systems producing a detectable $\gamma$-ray flux above $1 \mathrm{TeV}$ are prime candidates to also be detectable neutrino sources (see below).

\section{SOURCE LOCATION AND LUMINOSITY}

The flux expected at Earth from an isolated star can be computed as $F_{\gamma}\left(E_{\gamma}>1 \mathrm{TeV}\right)=\left(1 / 4 \pi D^{2}\right) \int_{R_{*}}^{R_{\text {wind }}} \int_{1 \mathrm{TeV}} n(r) q_{\gamma}\left(E_{\gamma}\right) \times$ $4 \pi r^{2} d r d E_{\gamma}$. The models in Table 1 , at $2 \mathrm{kpc}$, give fluxes in the range $\left(1 \times 10^{-20}\right.$ to $\left.7 \times 10^{-16}\right) K_{p}$ photons $\mathrm{cm}^{-2} \mathrm{~s}^{-1}$. Hence, there are models for which a small group of $\sim 10$ stars in a region 
with a CR enhancement factor of $\sim 100$ might be detectable at the level of $\sim 10^{-14}$ photons $\mathrm{cm}^{-2} \mathrm{~s}^{-1}$.

CRs are expected to be accelerated in $\mathrm{OB}$ associations through turbulent motions and collective effects of stellar winds (e.g., Bykov \& Fleishman 1992a, 1992b). The main acceleration region for $\mathrm{TeV}$ particles would be in the outer boundary of the supperbubble produced by the core of the association. If there is a subgroup of stars located at the acceleration region, their winds might be illuminated by the locally accelerated protons, which would have a distribution with a slope close to the canonical value, $\alpha \sim 2$. For stars out of the acceleration region, the changes introduced in the proton distribution by the diffusion of the particles (a steepening of its spectrum) would render the mechanism for $\mathrm{TeV} \gamma$-ray production inefficient. This can be seen from Table 1 through the strong dependency of the predicted $\mathrm{TeV}$ luminosity on the spectral slope of the particles.

An important assumption in our model is that the diffusion coefficient is a linear function of the particle energy in the inner wind. This is indeed an assumption also in both Völk \& Forman (1982) and White's (1985) models of the particle diffusion in the strong winds of early-type stars, among other studies. Measurements of the solar wind, however, seem to suggest a harder relation with energy (e.g., $D \propto E^{0.4-0.5}$; Ginzburg \& Syrovatskii 1964 , p. 336). If such a relation would hold for the inner wind of an $\mathrm{O}$ star (where $p p$ interactions occur), depending on the constant of proportionality, it could yield a higher value of $E_{p}^{\mathrm{min}}$ and hence a lower $\gamma$-ray luminosity. However, contrary to what happens with the Sun, in early-type stars line-driven instabilities are expected to produce strong shocks in the inner wind (Lamers \& Cassinelli 1999). In such a scenario, as emphasized by White (1985), to expect that particles will diffuse according to the Bohm parameterization seems not to be unreasonable. As we discuss in the next section, direct observation of $\mathrm{TeV}$ sources of stellar origin can shed light on the issue.

\section{APPLICATION: THE UNIDENTIFIED TeV SOURCE}

The High Energy Gamma Ray Astronomy (HEGRA) detection in the vicinity of Cygnus OB2, TeV J2032+4131 (Aharonian et al. 2002), presents an integral flux $F_{\gamma}\left(E_{\gamma}>1 \mathrm{TeV}\right)=$ $4.5( \pm 1.3) \times 10^{-13}$ photons $\mathrm{cm}^{-2} \mathrm{~s}^{-1}$ and a $\gamma$-ray spectrum $F_{\gamma}\left(E_{\gamma}\right)=B\left(E_{\gamma} / \mathrm{TeV}\right)^{-\Gamma}$ photons $\mathrm{cm}^{-2} \mathrm{~s}^{-1} \mathrm{TeV}^{-1}$, where $B=$ $4.7\left( \pm 2.1_{\text {stat }} \pm 1.3_{\text {sys }}\right) \times 10^{-13}$ and $\Gamma=1.9\left( \pm 0.3_{\text {stat }} \pm 0.3_{\text {sys }}\right)$. No counterparts at lower energies are currently identified (Butt et al. 2003; Mukherjee et al. 2003). The source flux was constant during the $3 \mathrm{yr}$ of data collection. The extension of the source $(5.6 \pm 1$ '.7) disfavors a pulsar or active galactic nuclei origin. The absence of an X-ray counterpart additionally disfavors a microquasar origin. Instead, the location of the $\mathrm{TeV}$ source, separate from the core of the association and coincident with a significant enhancement of the star number density (see Fig. 1 of Butt et al. 2003), might suggest the scenario outlined in the previous section.

A nearby EGRET source is, on the other hand, coincident with the center of the association, where it might be produced in the terminal shocks of powerful stars therein existing (White \& Chen 1992; Chen et al. 1996), in the colliding wind binary system Cyg OB2 5 (Benaglia et al. 2001), or in a combination of these scenarios. Contributions from the inner winds of $\mathrm{OB}$ stars as in the model herein explored cannot be ruled out. These, however, are not expected to dominate because of wind modulation (at low energies) and because of the softening of the CR spectrum while diffusing from the superbubble accelerating region, which significantly diminishes the number of $p-p$ interactions in the winds.

Our model could explain the unidentified TeV source without requirements other than the presence of the already observed stars and a reasonable CR enhancement if the density of the original ISM was rather low. Butt et al. (2003) argued for a density of $n_{0}=30 \mathrm{~cm}^{-3}$. However, this should be taken as a generous upper limit: (1) Apparently, there is no star formation currently active at the position of the source. (2) The CO- $\mathrm{H}_{2}$ conversion factor used to compute the density has been taken as the normal Galactic one, but it could be lower in the neighborhood of star-forming environments (e.g., Yao et al. 2003). (3) The particle density within the TeV source region has been averaged from a velocity range integrated along the line of sight corresponding to $3700 \mathrm{pc}$ and including the core of the Cygnus association. (4) The TeV source will actually be immersed in the zone 2 of the winds of the several powerful stars therein detected, which should have swept the ISM away and diminished its density. Our models (e.g., model g of Table 1), which in fact take for the stellar parameters an average value from the stars in Table 3 of Butt et al. (2003), show that the illumination of the innermost regions of the winds of $\sim 10$ stars with a CR enhancement of $\sim 300$ in a medium density of about $0.1 \mathrm{~cm}^{-3}$ may be enough to produce the HEGRA detection. The neutrino flux that results from a hadronic production of the $\mathrm{TeV} \gamma$-ray source would not produce a significant detection in AMANDA II, which is consistent with the latest reports by the AMANDA collaboration (Ahrens et al. 2003). In ICECUBE, however, the signal-to-noise ratio is $\sim 1.8$ for $1 \mathrm{yr}$ of observation (for energies above $1 \mathrm{TeV}$, an effective area of $1 \mathrm{~km}^{2}$, before taking into account neutrino oscillations effects). If ICECUBE can reach a $1^{\circ} \times 1^{\circ}$ or finer search bin and a $\mathrm{km}^{2}$ effective area at $\mathrm{TeV}$ energies, a long integrating time could distinguish the hadronic origin of the HEGRA detection.

\section{CONCLUDING REMARKS}

Hadronic interactions within the innermost region of the winds of $\mathrm{O}$ and $\mathrm{B}$ stars can produce significant $\gamma$-ray luminosities at $\mathrm{TeV}$ energies, with low brightness at other energies. At distances less than a few kiloparsecs, several illuminated winds pertaining to subgroups of stars located at CR acceleration regions in $\mathrm{OB}$ associations might be detected by Cerenkov telescopes. A reasonable set of model parameters can be found to produce a flux compatible with the only unidentified $\mathrm{TeV}$ source known. A candidate selection for possible new $\mathrm{TeV}$ sources, based on these predictions, will be reported elsewhere.

We thank L. Anchordoqui, P. Benaglia, Y. Butt, C. Mauche, F. Miniatti, R. Porrata, and H. Völk for useful discussions. The work of D. F. T. was performed under the auspices of the US DOE (NNSA), by UC's LLNL under contract W-7405-Eng-48. E. D.-S. acknowledges the Ministry of Science and Technology of Spain for financial support and the IGPP/LLNL for hospitality. G. E. R. is supported mainly by Fundación Antorchas and, in addition, from grants PICT 03-04881 (ANPCyT) and PIP 0438/ 98 (CONICET). He is grateful to the Hong Kong University and Professor K. S. Cheng for hospitality. 


\section{REFERENCES}

Aharonian, F. A., \& Atoyan, A. M. 1996, A\&A, 309, 917

Aharonian, F. A., et al. 2002, A\&A, 393, L37

Ahrens, J., et al. 2003, Phys. Rev. Lett., submitted (astro-ph/0309585)

Alvarez-Muñiz, J., \& Halzen, F. 2002, ApJ, 576, L33

Anchordoqui, L. A., et al. 2003, ApJ, 589, 481

Benaglia, P., Romero, G. E., Stevens, I. R., \& Torres, D. F. 2001, A\&A, 366, 605

Butt, Y. M., et al. 2003, ApJ, 597, 494

Bykov, A. M. 2001, Space Sci. Rev., 99, 317

Bykov, A. M., \& Fleishman, G. D. 1992a, MNRAS, 255, 269

1992b, Soviet Astron. Lett., 18, 95

Cassé, M., \& Paul, J. A. 1980, ApJ, 237, 236

Castor, J., McCray, R., \& Weaver, R. 1975, ApJ, 200, L107

Chen, W., White, R. L., \& Bertsch, D. 1996, A\&AS, 120, 423

Cox, A. N. 1999, Allen's Astrophysical Quantities (New York: Springer)

Dermer, C. D. 1986, A\&A, 157, 223

Donati, J.-F., et al. 2002, MNRAS, 333, 55 2001, MNRAS, 326, 1265

Drury, L., O'C., Aharonian, F. A., \& Völk, H. J. 1994, A\&A, 287, 959

Gaisser, T. K., Halzen, F., \& Stanev, T. 1995, Phys. Rep., 258, 173 (erratum 271, 355 [1996])

Ginzburg, V. L., \& Syrovatskii, S. I. 1964, The Origin of Cosmic Rays (New York: Pergamon)
Jokipii, J. R., Kóta, J., \& Merényi, E. 1993, ApJ, 405, 782

Jokipii, J. R., \& Parker, E. N. 1970, ApJ, 160, 735

Karle, A. 2002, preprint (astro-ph/0209556)

Kóta, J., \& Jokipii, J. R. 1983, ApJ, 265, 573

Lamers, H. J. G. L. M., \& Cassinelli, J. P. 1999, Introduction to Stellar Winds (Cambridge: Cambridge Univ. Press)

Lipari, P. 1993, Astropart. Phys., 1, 195

Montmerle, T. 1979, ApJ, 231, 95

Mukherjee, R., et al. 2003, ApJ, 589, 487

Parker, E. M. 1958, Phys. Rev., 110, 1445

Romero, G. E., Benaglia, P., \& Torres, D. F. 1999, A\&A, 348, 868

Romero, G. E., \& Torres, D. F. 2003, ApJ, 586, L33

Romero, G. E., Torres, D. F., Kaufman-Bernadó, M. M., \& Mirabel, I. F. 2003, A\&A, 410, L1

Torres, D. F., et al. 2003, Phys. Rep., 382, 303

Völk, H. J., \& Forman, M. 1982, ApJ, 253, 188

Volkova, L. V. 1980, Soviet J. Nucl. Phys., 31, 784

Weber, E. J., \& Davis, L. 1967, ApJ, 148, 217

White, R. L. 1985, ApJ, 289, 698

White, R. L., \& Chen, W. 1992, ApJ, 387, L81

Yao, L., Seaquist, E. R., Kuno, N., \& Dunne, L. 2003, ApJ, 588, 771 